\title{
Computerized Angiographic Occlusion Rating for Ruptured Clipped Aneurysms is Superior to Subjective Occlusion Rating
}

\author{
(D)A.R. Al-Schameri, DG. Baltsavias, P. Winkler, M. Lunzer, DM. Kral, L. Machegger, F. Weymayr, S. Emich, C. Sherif, and B. Richling
}

\begin{abstract}
BACKGROUND AND PURPOSE: The computerized occlusion rating to estimate angiographic occlusion of embolized aneurysms is superior to the subjective occlusion rating. In this study, we compared the 2 methods in the analysis of aneurysms clipped after subarachnoid hemorrhage.
\end{abstract}

MATERIALS AND METHODS: The pre- and postoperative angiographic images (DSA) of 95 selected patients were analyzed and stratified in 4 grades (grade 0 for $100 \%$, grade I for $<99 \%-90 \%$, grade II for $<89 \%-70 \%$, grade III for $<70 \%$ occlusion) by using the subjective (angiographic) occlusion rating and the computerized (angiographic) occlusion rating. For the subjective occlusion rating, the occlusion rate was estimated; for the computerized occlusion rating, the "occluded" and "nonoccluded" aneurysm areas were automatically calculated in square millimeters after outlining the ideal occlusion line.

RESULTS: With the subjective occlusion rating, 75 (78.9\%), 12 (12.6\%), 7 (7.4\%), and 1 (1.1\%) and with the computerized occlusion rating 45 (47.4\%), 24 (25.3\%), 20 (21.0\%), and 6 (6.3\%) patients had aneurysms stratified to grades 0 , I, II and III, respectively. The interobserver variation was significant with the subjective occlusion rating but not with the computerized occlusion rating. The subjective occlusion rating overestimated aneurysm occlusion in $30(31.6 \%$ ) patients. Mean values were the following: subjective occlusion rating of $97.5 \pm 6.3 \%$ and computerized occlusion rating of $93.5 \pm 9.7 \% ; P=<.001$. No patient rebled, and 4 patients underwent retreatment during $36 \pm 38.9$ months; the predictive value (log-rank, Kaplan-Meier) of the subjective and computerized occlusion ratings with respect to retreatment was highly significant for both methods (subjective occlusion rating: $\chi^{2}, 29.65 ; P<.001$; computerized occlusion rating: $\chi^{2}, 35.57, P<.001$ ).

CONCLUSIONS: The 2 methods showed remarkable differences in the estimation of the angiographic occlusion rates of clipped aneurysms. The clearly lower interobserver variation of the computerized versus subjective occlusion rating may indicate a superiority of the computerized occlusion rating.

ABBREVIATIONS: COR = computerized (angiographic) occlusion rating; SOR = subjective (angiographic) occlusion rating

A lthough endovascular embolization is now seen as the firstline treatment for most ruptured intracranial aneurysms, a significant percentage of patients have undergone microsurgical clipping. ${ }^{1}$ The main advantage of aneurysm clipping is its potential ability to occlude aneurysms totally and permanently. ${ }^{2}$ However, failures in clip placement or stability, due to misinterpreta-

Received November 27, 2014; accepted after revision February 14, 2015.

From the Departments of Neurosurgery (A.R.A.-S., P.W., M.L., M.K., S.E., B.R.), Neuroradiology (L.M., F.W.), Paracelsus Private Medical University, Salzburg, Austria; Department of Neuroradiology (G.B.), University Hospitals of Zurich, Zurich, Switzerland; and Department of Neurosurgery (C.S.), Krankenanstalt Rudolfstiftung, Vienna, Austria.

Please address correspondence to A.R. Al-Schameri, MD, Department of Neurosurgery, Paracelsus Private Medical University, Salzburg, Ignaz Harrer Str 79, 5020 Salzburg, Austria; e-mail: a.al-schameri@salk.at

http://dx.doi.org/10.3174/ajnr.A4399 tion of angiograms or surgical findings, may lead to early or late development of aneurysm remnants, possibly causing recurrent hemorrhage. ${ }^{3,4}$ Aneurysm remnants are evaluated and followed up mostly by angiography. Multiple attempts have been made to categorize the great variety of different types of aneurysm remnants, ${ }^{2,5-8}$ but only a few articles have been published concerning the angiographic assessment details being used to delineate and quantify aneurysm remnants after aneurysm clipping. ${ }^{2,4,9}$ All authors based the angiographic assessment on the criterion standard of the subjective occlusion rating (SOR). We recently advocated the superiority of the computerized angiographic occlusion rating (COR) versus subjective occlusion estimations for ruptured embolized aneurysms, due to reduction of subjective estimation bias and interobserver variations. ${ }^{10}$ In the present study, COR was retrospectively applied to a series of 95 patients who underwent aneurysm clipping after subarachnoid hemorrhage. 
Table 1: Patient characteristics

\begin{tabular}{lc}
\hline \multicolumn{1}{c}{ Characteristics } & \\
\hline Patients (No.) & 95 \\
Mean age (yr) & 49 \\
Male sex (No.) & 31 \\
Female sex (No.) & 64 \\
Mean time of follow-up (mo) & 34,21 \\
Intraoperative rupture & 3 \\
\hline
\end{tabular}

Table 2: Baseline parameters

\begin{tabular}{lccccc}
\multicolumn{1}{c}{ Grading } & $\mathbf{1}$ & $\mathbf{2}$ & $\mathbf{3}$ & $\mathbf{4}$ & $\mathbf{5}$ \\
\hline Fisher (No.) & 15 & 37 & 24 & 19 & \\
Hunt and Hess (No.) & 53 & 23 & 14 & 3 & 2 \\
\hline
\end{tabular}

Table 3: Aneurysm location

\begin{tabular}{lr}
\hline \multicolumn{1}{c}{ Location } & No. \\
\hline Anterior cerebral artery & 31 \\
Pericallosal artery & 9 \\
Internal carotid artery & 9 \\
Middle cerebral artery & 38 \\
Posterior communicating artery & 4 \\
Posterior circulation & 4 \\
\hline
\end{tabular}

\section{MATERIALS AND METHODS \\ Patients}

From a series of 671 patients treated for aneurysms at the Department of Neurosurgery, Paracelsus Private Medical University of Salzburg, from July 1998 until December 2012, 340 patients underwent clipping. Of those, 232 patients with 310 aneurysms had a subarachnoid hemorrhage. All patients were operated on by using standard microsurgical techniques; temporary clipping was applied on 12 cases. We used Aesculap ${ }^{\circledR}$ Yasargil clips (B.Braun, Tuttlingen, Germany) in all cases. From these 232 patients, 137 were excluded due to either death before follow-up angiography ( $n=54)$ or loss to follow-up or missing data $(n=83)$. Therefore, 95 patients were included for analysis. At the time of the operation (mean, 3 days after hemorrhage; maximum/minimum, 37/0 days), 76 patients were Hunt and Hess grade 1-2, 14 patients were Hunt and Hess grade 3, and 5 were Hunt and Hess grades 4-5 (Tables 1-3). Patient data were retrospectively entered into a computerized data base. ${ }^{10}$ From this data base, we extracted demographic data (name, age, sex), aneurysm location, Fisher grading, operation date, special circumstances of surgery, and follow-up data (outcome scores, angiographic results, and retreatments).

\section{Angiographic Image Selection}

In this study, we included only patients with available pre- and postoperative and follow-up DSA examinations; each of the selected images was taken at the phase of maximum arterial perfusion. Oblique DSA projections were excluded due to inferior comparability between pre- and postoperative projections, introducing bias for later image superimposition used in COR. For SOR, 2 experienced neuroradiologists not involved in the aneurysm treatment and blinded to the patient outcome, independently evaluated the angiographic material to minimize bias caused by image selection and interpretation. For COR, 2 independent neurosurgeons not involved in the aneurysm treatment and blinded to the patient outcome assessed the true anteroposterior and lateral views, guaranteeing ideal later image fusion. Cases of disagreement between the 2 investigators of each assessment technique were settled by consensus.

\section{Subjective Occlusion Rating}

To estimate SOR, we had to choose noncontinuous parameters. In our previous article on coiled aneurysms, ${ }^{10} \mathrm{SOR}$ was estimated as $100 \%, 95 \%, 90 \%, 80 \%, 70 \%$, and $60 \%$. For clipped aneurysms, the estimation of SOR was even more difficult because the occluded aneurysm sac is invisible on postsurgical DSA. We thus defined larger intervals for low-occluded aneurysms (100\%, 95\%, $80 \%, 60 \%)$. These percentage values were used for statistical evaluations and comparisons.

After SOR was estimated by the 2 reviewers, the SOR percentage values were assigned for better comparability with the neurosurgical literature to a modified version of the classification proposed by Sindou et $\mathrm{al}^{2}$ as follows: Grade 0 was added, representing $100 \%$ occlusion; grade I (range, 99.9\%-90\%) was assigned to SOR $95 \%$, grade II (range, $89.9 \%-70 \%$ ) was assigned to SOR $80 \%$, and grade III ( $<70 \%)$ was assigned to $60 \%$ occlusion because we did not observe any lower SOR estimations in this series.

\section{Computerized Occlusion Rating}

For COR, the previously published techniques by Sherif et $\mathrm{al}^{10}$ had to be modified for clipped aneurysms: In coiled aneurysms, the parameters "recanalized area" and "total aneurysm area" can be easily defined on the postembolization angiograms alone because the coils are visible on the native angiographs. However, on postoperative angiographs of clipped aneurysms, the occluded aneurysm sac is always invisible. This problem was solved by superimposition of preoperative and postoperative angiograms. This was feasible because for angiography, we were always careful on precise anteroposterior and lateral projections. For superimposition, the custom-made software Control-2D (NV Tec Neurovascular Technologies, Vienna, Austria) was used. It allows DSA image superimposition with high precision, resulting in a single angiographic image disclosing the clip with or without an aneurysm remnant. After the 2 reviewers had outlined the orifice plane ("missing vessel wall" or "ideal occlusion line") and the actual occlusion line given by the clips, the following parameters were measured automatically (Fig 1):

1) Total aneurysm area (square millimeters): Borders of the contrast-filled aneurysm and the defined orifice plane (ideal occlusion line) were outlined and the area was calculated.

2) Aneurysm nonoccluded area (square millimeters): The area filled by contrast medium outside the defined orifice plane (ideal occlusion line) was calculated as "nonoccluded area."

3) Finally, the occluded area of the aneurysm was calculated as the difference of "total area" minus "nonoccluded area" and was given as a percentage core (to allow direct comparison with the SOR values).

\section{Statistics}

Interobserver variability was calculated for SOR and COR by using the Wilcoxon test.

For every patient, the mean values of both observers were cal- 

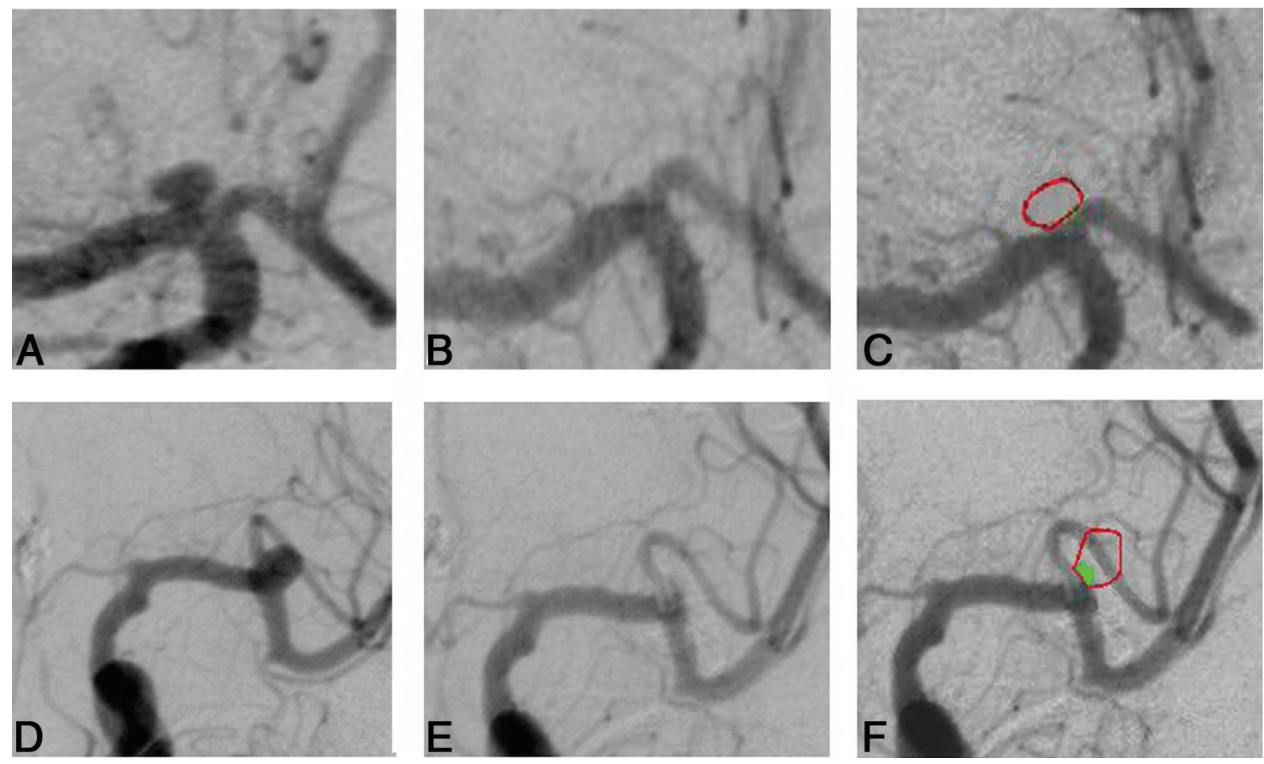

FIG 1. Case 1: $A$, disclosing ICA bifurcation aneurysm in ap projection; $B$, after clipping; $C$, superimposition of $B$ and $C$; no remnant. Case 2: $D$, disclosing $\mathrm{M} 1 / \mathrm{M} 2$ aneurysm in ap projection; $E$, after clipping; and $F$, shows superimposition of $D$ and $E$, the outlined aneurysm (red) and the remnant (green).

Table 4: SOR and COR results

\begin{tabular}{lccccc}
\hline & $\mathbf{0}(\mathbf{1 0 0 \% )}$ & I (99.9\%-90\%) & II (89.9\%-70\%) & III (<70\%) & \\
\hline SOR pts (\%) & $75(78.9 \%)$ & $12.6(12.6 \%)$ & $7(7.4 \%)$ & $1(1.1 \%)$ & $95 / 100 \%$ \\
COR pts (\%) & $45(47.4 \%)$ & $24(25.3 \%)$ & $20(21.0 \%)$ & $6(6.3 \%)$ & $95 / 100 \%$ \\
\hline
\end{tabular}

Note:- pts indicates patients.

\begin{tabular}{|c|c|c|c|c|c|}
\hline Modality/Group & 0 & I & II & III & Total Nr. \\
\hline SOR & 75 & 12 & 7 & 1 & 95 \\
\hline COR & 45 & 24 & 20 & 6 & 95 \\
\hline Diff. strat. & 30 & 15 & 13 & 2 & \\
\hline
\end{tabular}

Note:-Diff. strat. indicates patients differently stratified.

Table 6: Stratification of the 4 retreated patients by the 2 SOR and 2 COR observers

\begin{tabular}{lcccc}
\hline & SOR 1 & SOR 2 & COR 1 & COR 2 \\
\hline Pt 1 & 0 & I & II & II \\
Pt 2 & II & III & III & III \\
Pt 3 & I & II & III & III \\
Pt 4 & 0 & 0 & I & I \\
\hline
\end{tabular}

Note:-Pt indicates patient.

culated for SOR and COR. Then COR and SOR, classified as $100 \%, 99.9 \%-90 \%, 89.9 \%-70 \%$, and $<70 \%$, were evaluated as predictors of outcome in univariate Kaplan-Meier analysis (logrank test) with censoring at the time of retreatment. SOR and COR were compared by using the paired $t$ test. Data are presented as mean $\pm \mathrm{SD}$. A $P$ value $<.05$ was considered significant. SAS 8.02 (SAS Institute, Cary, North Carolina) software was used for all statistical calculations.

\section{RESULTS}

The angiographic follow-up was $13.5 \pm 18.7$ months; the clinical follow-up was $34.0 \pm 38.9$ months. A consensus evaluation of selected DSA projections was necessary in 5 patients (5.3\%). Statistically significant interobserver variation was documented for SOR measurements $(P=.002)$. For COR, the interobserver vari- ation was not significant $(P=1,35)$. When using the SOR method, 75 (78.9\%) patients had aneurysms stratified to grade 0 ( $100 \%$ occlusion), compared with only $45(47.4 \%)$ patients when using the COR technique. To grade I (99.9\%-90\% occlusion), 12 (12.6\%) patients were stratified by using SOR technique, compared with 24 (25.3\%) by using the COR method. To grade II ( $89.9 \%-70 \%$ occlusion), 7 patients (7.4\%) were stratified by using SOR, but $20(21.0 \%)$, by using COR. Finally to grade III ( $<70 \%$ occlusion), 1 patient $(1,1 \%)$ was stratified when using SOR, but 6 patients $(6,3 \%)$, when using the COR method (Table 4).

When we compared the values of SOR and COR, the SOR method overestimated the degree of aneurysm occlusion in 30 $(31.6 \%)$ patients (Table 5$)$. The mean value of SOR was $97.5 \pm$ $6.3 \%$, and of COR, $93.5 \pm 9.7 \%(P=<.001)$. In the clinical follow-up (mean, 34 months), no patient rebled. Four patients required retreatment. Of those, 2 patients were categorized into grade 0 by using SOR; by using COR, these 2 patients were stratified to grade I. The other 2 patients were stratified by the 2 SOR observers to different grades (I, II, and III) with high interobserver variation, whereas COR classified them homogeneously to grade III (Table 6). The predictive value (log-rank, Kaplan-Meier) of SOR and COR with respect to retreatment was highly significant for both methods (SOR: $\chi^{2}, 29.65, P<.0001$; COR: $\chi^{2}, 35.57, P<$ $.0001)$.

\section{DISCUSSION}

In the past, outcome studies after aneurysm treatment were presented even without follow-up angiography. ${ }^{11}$ In the surgical arm of the Cerebral Aneurysm Rerupture After Treatment study, ${ }^{11}$ representing $70.6 \%$ of the total study population, aneurysm occlusion was estimated by the surgeon after clipping in those cases in which DSA was not available. Today conventional angiography 
remains not only the main postoperative diagnostic tool ${ }^{2,3,6,8,12}$ but also the most reliable for late follow-up. The aim of angiography after aneurysm clipping is, besides the detection of unclipped aneurysms or occluded vessels, ${ }^{7}$ the verification of aneurysm remnants. This allows planning of follow-up intervals and retreatment and the prediction of rebleeding risks..$^{2,3,5,7,11,12}$ Because the goal of surgical aneurysm therapy is total and permanent occlusion of the aneurysm, partial occlusion has been seen as a failure due to the potential consequences of recurrent aneurysm hemorrhage. ${ }^{3,11}$ Factors like the size and shape of the reperfused aneurysm, the relation of the open aneurysm part to the parent vessel, and the challenge of predicting the risk of rebleeding have been widely discussed in the literature. ${ }^{8,11,13,14}$ Currently, the standard method in the assessment of the occlusion rate of a clipped aneurysm is a subjective occlusion rating based on postoperative early or late angiography. However, recently we showed that for ruptured coil-embolized cerebral aneurysms, the computerized occlusion rating is superior to the SOR by reducing subjective bias in estimating occlusion rates and by avoiding interobserver variability. ${ }^{10}$ The technical differences between the occlusion of an aneurysm by coils compared with clips and the different angiographic appearance of the occluding material made it necessary to modify the initial software. Thus, the possibility of image superimposition was improved. Perfect image superimposition is mandatory for COR of clipped aneurysms because the occluded part of the aneurysm is invisible.

Because the initial occlusion rate is the most important parameter for follow-up strategies, the method of occlusion rating should be reproducible and objective. Therefore, we assessed interobserver variations of SOR and COR. The statistically significant interobserver variation for COR demonstrates that subjective bias may falsify the results of angiographic assessments and lead to incorrect follow-up or treatment decisions for patients. When further comparing SOR with COR, we found a different stratification of $31.6 \%$ of all patients to a higher occlusion rate grade. Thus, from 75 patients assigned to grade 0 by SOR, 26 were stratified to grade II; and 4 , to grade III by COR (Table 5).

Neither of the 2 methods could validate the results. Both methods are (in this study) based on angiographic pictures taken in strict anteroposterior and lateral projections. With SOR, only assessments can be achieved, whereas with COR, a quantification of the "nonoccluded area" is given in square millimeters. An analysis of 3D structures must be obtained to approach a validation (see below), but because the results will still rely on angiographic pictures, true validations cannot be expected.

\section{Total Aneurysm Occlusion}

Of special note, the rate of "total occlusion," commonly seen as an important parameter expressing the quality of aneurysm surgery, was $79 \%$ by using SOR, but only $47.4 \%$ with COR. Comparing the high number of "misclippings" (21\% with SOR) with literature data, one can find that of 5 publications dealing with angiographic clipping rates, 5 describe patients having both ruptured and unruptured aneurysms; there, the rates of misclipping are $4.4 \%-$ $18.6 \% .{ }^{15-18}$ The only report analyzing only patients clipped after subarachnoid hemorrhage as in the current study is the one by $\mathrm{Yu}$ et al, ${ }^{19}$ who, in 2007 , described a series of 169 patients with rup- tured aneurysms. The authors disclosed a misclipping rate of $16 \%$, but there was no comparable information about preoperative clinical grading in this study, because "patients in poor conditions warranted greater delay." 19 In our series, all patients had subarachnoid hemorrhage and 19 were medium or poor grade at the time of surgery (Tables 1-3). A further factor that may have influenced our misclipping rate is that in emergency cases, the operations have also been performed by neurosurgeons less experienced in aneurysm therapy. An overview of the experience level of the surgeons involved in our misclipped cases showed that more experienced surgeons had lower aneurysm remnant rates.

When we used COR, the rate of incompletely occluded aneurysms increased to $52.6 \%$. These unusual numbers must be seen in the light of the high capacity of the COR software to detect very small areas of contrast in the field of assessment, compared with the human eye. Due to the frequent mismatch between the straight or curved clip blade and the anatomy of the neck site, the angiogram eventually shows minimal amounts of contrast material outside the "ideal occlusion line," which was defined by the 2 investigating neurosurgeons. If the clips could not be applied parallel to the parent vessel (eg, rectangular or "palisade-like" clip application), the amount of extravasal dye may be even more. It is the responsibility of the surgeon to accept a certain mismatch or to improve his results by changing or adding clips, both steps eventually linked to an increased risk of an immediate complication, especially after previous subarachnoid hemorrhage. All these circumstances will finally influence the rate of misclipping.

\section{Retreatment}

In our series, we observed no recurrent hemorrhage, but 4 patients $(4.2 \%)$ underwent retreatment. When we looked at the stratification of the retreated patients, 2 patients were stratified by SOR to grade 0 , but by COR, to grade I. The former indication for retreatment of these 2 patients had been made by the surgical team by using the common SOR. These 2 different SOR assessments at 2 different points in time express the influence of subjective bias with SOR. Another patient stratified to the grades I/II by the 2 SOR investigators was stratified to grades III/III by using COR. The fourth patient was stratified to II/III by SOR and to III/III by COR. Three of 4 patients stratified by SOR showed high interobserver variation. For any retreated patient, the stratification between the 2 COR observers was equal (Table 6). Again this finding shows the high impact of subjective overestimation of aneurysm occlusion by SOR. It also demonstrates that despite statistically high predictive value (log-rank, Kaplan Meier), the clinical value of SOR may be limited by incorrect overestimation of aneurysm occlusion. With the COR method however, the degree of aneurysm occlusion was in accordance with the estimated need for retreatment in all cases.

The reasons for the differences between subjective and computerized assessments of contrast-perfused areas on angiograms are the following: the higher precision of the computer to calculate gray zones in square millimeters compared with an assessment by the human eye and the lack of accuracy by using anatomic structures to compare and define percentages of occlusion.

AJNR Am J Neuroradiol 36:1704-09 Sep 2015 www.ajnr.org 


\section{Clinical Impact}

The higher potential of COR to detect small zones of dye in anatomic areas belonging to the aneurysm may stimulate discussion about the clinical significance of "total occlusion" after aneurysm clipping because none of the 20 (SOR) or 50 (COR) patients with misclipping rebled. To define the minimum COR percentage to prevent retreatment and secondary hemorrhage, larger clinical studies are warranted. Nevertheless, COR provided reproducible measurements, allowing better comparison and showing a good relation to clinical parameters. SOR, on the contrary, has significant dependency on subjective bias with high interobserver variation and often an unclear relation of the results to clinical events.

\section{Limitations}

One limiting factor in the present study is its retrospective design with noncontinuous follow-up, and several patients were lost to follow-up for various reasons. Further limiting factors are the restriction to anteroposterior and lateral projections for angiography and the statistical comparison of the continuous parameter COR with the noncontinuous parameter SOR.

\section{Follow-Up}

To receive more information about rebleeding and retreatment, we analyzed those patients who were excluded from the study due to leakage on follow-up angiography. From the 77 patients in this additional group, no patient had a secondary hemorrhage or a retreatment; thus the overall retreatment rate was $2.3 \%$.

It seems realistic to assume a close relation between initial occlusion and retreatment. Because we observed enough end points to reach statistical significance despite the short follow-up, a longer follow-up could only show further end points, thus resulting in an even stronger relation. Nevertheless, our follow-up data represent a limitation in assessing potential delayed effects of incomplete, or even complete, aneurysm occlusion.

\section{Angiographic Projections}

Under ideal circumstances, oblique projections allowing good visualization of the aneurysm neck are available for pre- and posttreatment angiograms. Today, using angular projections is a routine procedure in endovascular aneurysm therapy because the rotational parameters of both $\mathrm{C}$-arms can be stored and reused for follow-up angiography. However, the retrospective nature of our study implied that for earlier cases of surgical therapy, oblique projections had been rarely used. If so, the repetition of an oblique projection at follow-up was either technically not possible, not seen as necessary, or performed by using nonidentical angles.

The limitations resulting from the sole use of straight projections are obvious and mainly concern the assessment of the shape and size of eventual aneurysm remnants. These limitations are equal for both methods. The primary goal of our study was to compare SOR and COR, both assessments based on the same angiographic projections and the same image content. We fully agree that oblique projections allow better assessment of aneurysm necks; the superiority of COR over SOR also improves the assessment of oblique comparative angiographic studies, as previously reported. ${ }^{20}$
In the future, COR can be used for 3D angiography of cerebral aneurysms, thus allowing not only increased precision of assessment but eventually also a volumetric quantification of aneurysm necks. A supplementary work by using 3D COR, which may elucidate this aspect, is in progress and will be reported.

\section{Statistical Comparison of SOR versus COR}

Only the use of noncontinuous parameters for SOR guarantees realistic approximations of occlusion rates and comparability in an everyday clinical setting. Despite statistical imprecision, it offers a realistic basis for comparison with the continuous-parameter COR.

\section{CONCLUSIONS}

The computerized angiographic occlusion rating is superior to the actual standard of subjective occlusion estimations. In addition, for clipped aneurysms, COR offers the possibility of improved quality control of the treatments by the use of the percentage of occlusion rating. It offers considerable reduction of subjective estimation bias and interobserver variations. SOR may lead to overestimations of occlusion rates, resulting in erroneous follow-up and retreatment strategies. The main factor limiting these results is the retrospective design of this study, with the consequence of reduced follow-up data and straight angiographic projections. The high sensitivity of COR in detecting minimal areas of contrast medium in the neck region should be confirmed in further studies by using 3D volumetric data.

Disclosures: Camillo Sherif-OTHER RELATIONSHIPS: shareholder of NVtec Neurovascular Technologies and CVtec Cerebrovascular Technologies.

\section{REFERENCES}

1. Molyneux AJ, Kerr RS, Yu LM, et al; International Subarachnoid Aneurysm Trial (ISAT) Collaborative Group. International subarachnoid aneurysm trial (ISAT) of neurosurgical clipping versus endovascular coiling in 2143 patients with ruptured intracranial aneurysms: a randomised comparison of effects on survival, dependency, seizures, rebleeding, subgroups, and aneurysm occlusion. Lancet 2005;366: 809-17

2. Sindou M, Acevedo JC, Turjman F. Aneurysmal remnants after microsurgical clipping: classification and results from a prospective angiographic study (in a consecutive series of 305 operated intracranial aneurysms). Acta Neurochir (Wien) 1998;140:1153-59

3. Wermer MJ, Rinkel GJ, Greebe P, et al. Late recurrence of subarachnoid hemorrhage after treatment for ruptured aneurysms: patient characteristics and outcomes. Neurosurgery 2005;56:197-204; discussion 197-204

4. Lee JH, Kim SJ, Cha J, et al. Postoperative multidetector computed tomography angiography after aneurysm clipping: comparison with digital subtraction angiography. J Comput Assist Tomogr 2005; 29:20-25

5. el-Beltagy M, Muroi C, Roth P, et al. Recurrent intracranial aneurysms after successful neck clipping. World Neurosurg 2010;74:472-77

6. David CA, Vishteh AG, Spetzler RF, et al. Late angiographic follow-up review of surgically treated aneurysms. J Neurosurg 1999;91:396-401

7. Wermer MJ, van der Schaaf IC, Velthuis BK, et al; ASTRA Study Group. Follow-up screening after subarachnoid haemorrhage: frequency and determinants of new aneurysms and enlargement of existing aneurysms. Brain 2005;128:2421-29

8. Tsutsumi K, Ueki K, Morita A, et al. Risk of aneurysm recurrence in 
patients with clipped cerebral aneurysms: results of long-term follow-up angiography. Stroke 2001;32:1191-94

9. Elijovich L, Higashida RT, Lawton MT, et al; Cerebral Aneurysm Rerupture After Treatment (CARAT) Investigators. Predictors and outcomes of intraprocedural rupture in patients treated for ruptured intracranial aneurysms: the CARAT study. Stroke 2008;39:1501-06

10. Sherif C, Gruber A, Schuster E, et al. Computerized occlusion rating: a superior predictor of aneurysm rebleeding for ruptured embolized aneurysms. AJNR Am J Neuroradiol 2012;33:1481-87

11. Johnston SC, Dowd CF, Higashida RT, et al; CARAT Investigators. Predictors of rehemorrhage after treatment of ruptured intracranial aneurysms: the Cerebral Aneurysm Rerupture After Treatment (CARAT) study. Stroke 2008;39:120-25

12. Thornton J, Bashir Q, Aletich VA, et al. What percentage of surgically clipped intracranial aneurysms have residual necks? Neurosurgery 2000;46:1294-98; discussion 1298-1300

13. Martin NA, Bentson J, Viñuela F, et al. Intraoperative digital subtraction angiography and the surgical treatment of intracranial aneurysms and vascular malformations. J Neurosurg 1990;73: 526-33

14. Rauzzino MJ, Quinn CM, Fisher WS. Angiography after aneurysm surgery: indications for "selective" angiography. Surg Neurol 1998; 49:32-40; discussion 40-41
15. Lot G, Houdart E, Cophignon J, et al. Combined management of intracranial aneurysms by surgical and endovascular treatment: modalities and results from a series of 395 cases. Acta Neurochir (Wien) 1999;141:557-62

16. Taha MM, Nakahara I, Higashi T, et al. Endovascular embolization vs surgical clipping in treatment of cerebral aneurysms: morbidity and mortality with short-term outcome. Surg Neurol 2006;66:27784; discussion 284

17. Ogilvy CS, Hoh BL, Singer RJ, et al. Clinical and radiographic outcome in the management of posterior circulation aneurysms by use of direct surgical or endovascular techniques. Neurosurgery 2002;51: 14-21; discussion 21-22

18. Murphy M, Bell D, Worth RD, et al. Angiography postclipping and coiling of cerebral aneurysms. Br J Neurosurg 2005;19:225-28

19. Yu SC, Wong GK, Wong JK, et al. Endovascular coiling versus neurosurgical clipping for ruptured intracranial aneurysms: significant benefits in clinical outcome and reduced consumption of hospital resources in Hong Kong Chinese patients. Hong Kong Med J 2007;13:271-78

20. Sherif C, Marbacher S, Fandino J, et al. 3D computerized occlusion rating of embolized experimental aneurysms using noninvasive 1.5T MR imaging. AJNR Am J Neuroradiol 2012;33:661-66 